\title{
Local Structure of Nitrogen Atoms in a Porphine Ring of meso- Phenyl Substituted Porphyrin with an Electron-Withdrawing Group Using X-ray Photoelectron Spectroscopy and X-ray Absorption Spectroscopy
}

\author{
Hisao Yamashige,* Shuji Matsuo, ** Tsutomu Kurisaki,* Rupert C. C. Perera,*** and \\ Hisanobu WAKITA*,**† \\ *Department of Chemistry, Faculty of Science, Fukuoka University, \\ 8-19-1 Nanakuma, Jonan-ku, Fukuoka 814-0180, Japan \\ **Advanced Materials Institute, Fukuoka University, 8-19-1 Nanakuma, Jonan-ku, Fukuoka 814-0180, Japan \\ ***Center for X-Ray Optics, Lawrence Berkeley National Laboratory, Berkeley, CA 94720, USA
}

\begin{abstract}
This study investigated the protonation of nitrogen atoms in porphyrins with meso-phenyl $p$-substituted by an electronwithdrawing group using $\mathrm{N}$ 1s X-ray photoelectron spectroscopy (XPS), the N K X-ray absorption near-edge structure (XANES), and the discrete variational (DV)-X $\alpha$ molecular orbital (MO) method. Both tetraphenylporphyrin (TPP) and tetrakis( $p$-sulfonatophenyl)porphyrin (TSPP) have a single structure: the former has two protonated and two nonprotonated $\mathrm{N}$ atoms in the porphine ring; the latter has four protonated $\mathrm{N}$ atoms in the porphine ring. In contrast, a combination of XPS, XANES, and DV-X $\alpha$ MO calculations shows that tetrakis( $p$-carboxyphenyl)porphyrin (TCPP) has a dual structure: one structure has two protonated and two non-protonated $\mathrm{N}$ atoms; the other has four protonated $\mathrm{N}$ atoms. Furthermore, this result was also considered based on the protonation constants of $\mathrm{N}$ atoms in the porphyrins. The correlation between the strength of electron-withdrawing groups and protonation to $\mathrm{N}$ atoms in porphyrins can be described using the spectral patterns of the N 1s XPS and N K XANES spectra.
\end{abstract}

(Received February 18, 2005; Accepted April 25, 2005)

\section{Introduction}

Metalloporphyrins with electron-withdrawing substitutes, such as carboxyl groups, at the meso-positions, and those immobilized on inorganic supports act as efficient and selective catalysts for the oxidation of hydrocarbons. ${ }^{1-7}$ Porphyrin ligands with electron-withdrawing substitutes (PPEW) are currently of interest as precursors to stable metalloporphyrin catalysts for a variety of hydrocarbon-oxygenation reactions that are important for future energy development. ${ }^{8-19}$ The effect of electronwithdrawing groups on the electron states of porphyrin macrocycles, especially of four nitrogen atoms in the porphine ring, is an important issue for studies in not only analytical chemistry, but also in coordination chemistry because it is related to the protonation of nitrogen atoms in the porphine ring and to the stability of metalloporphyrin complexes.

Changes of the local structure and electronic states between four nitrogen atoms and protons in the porphine ring in the PPEW ligand have been elucidated using $a b$ initio calculations ${ }^{20}$ and ionization potentials. The results of local-density functional calculations and spectroscopic studies show that they are different from the meso and $\beta$ positions of a variety of multiple substituents (alkyl-, aryl-, fluoro-, chloro-, bromo-, trifluoromethyl cyano-, nitro-, etc.) in the PPEW that were provided. ${ }^{21}$ However, information regarding molecular orbitals

$\doteqdot$ To whom correspondence should be addressed.

E-mail: wakita@fukuoka-u.ac.jp
(MOs), including the atomic orbitals of nitrogen in these literatures was not investigated. On the other hand, because the effect of electron-withdrawing groups strongly affects the energy level of the $1 \mathrm{~s}$ orbital for nitrogen, it is necessary to reveal the electronic effect. Therefore, X-ray photoelectron spectroscopy (XPS) is a very suitable method because it is possible to observe the atomic orbital with a characteristic energy. In addition, the X-ray absorption near-edge structure (XANES) provides an effective verification of the local structure of an absorbing atom. So far, a correlation between the energy of the $1 \mathrm{~s}$ orbital for $\mathrm{N}$ in a porphine ring and the electron-withdrawing strength of meso-substituents in the PPEW has been reported to be a result of XPS and $a b$ initio calculations. ${ }^{22}$ On the other hand, N K XANES measurements of porphyrins have been reported for the orientation and electronic structure of macromolecular arrays containing porphyrins, ${ }^{23}$ porphyrin thin film, ${ }^{24}$ porphyrins in petroleum asphaltenes, ${ }^{25}$ and porphyrins with adsorbed nitrogen monoxide. ${ }^{26}$

Nevertheless, few studies have used a combination of XPS and XANES, a correlation between the protonation to nitrogen atoms in a porphine ring, and the strength of electronwithdrawing groups in a meso-substituted porphyrin. To show correlations in this study, the spectra of $\mathrm{N} 1 \mathrm{~s}$ XPS and N K XANES for tetrakis(p-carboxyphenyl)porphyrin (TCPP) were measured and analyzed from an electronic state calculation using the discrete variational (DV)-X $\alpha \mathrm{MO}$ method, ${ }^{27}$ which is a first-principle calculation that is useful for the theoretical analysis of X-ray spectroscopies. ${ }^{28}$ The spectra of N 1s XPS 


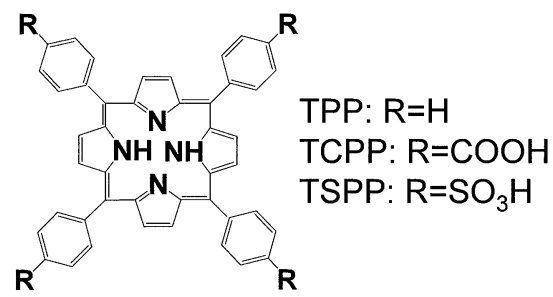

Fig. 1 Structure of meso-phenyl substituted (R) porphyrin: TPP, TSPP, and TCPP are $\mathrm{R}=\mathrm{H}, \mathrm{SO}_{3} \mathrm{H}$, and $\mathrm{COOH}$, respectively.

and N K XANES for tetraphenylporphyrin (TPP) and tetrakis( $p$ sulfonatophenyl)porphyrin (TSPP) were also measured as references. Their numbers of protonated nitrogen atoms are known to be two and four, respectively.

\section{Experimental}

\section{Materials}

Porphyrins (see Fig. 1) were purchased from different manufacturers: TPP (> 99\%; Sigma-Aldrich Corp., Missouri, U.S.), TCPP (> 97\%; Frontier Scientific Corp., Utah, U.S.), and TSPP (> 98\%; Dojindo Laboratories, Kumamoto, Japan). They were used for XPS and XANES measurements without further purification.

\section{$X P S$}

We carried out XPS measurements using a spectrometer (PHI

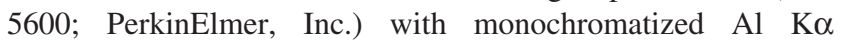
radiation. The X-ray source was operated at $14 \mathrm{kV}$ and $400 \mathrm{~W}$. The instrument contained a hemispherical analyzer with apertures for spot sizes of $800 \mu \mathrm{m}$. The angle of the photon incidence was varied from 0 to $45^{\circ}$; survey and high-resolution spectra were collected with step energies of 0.8 at $0.2 \mathrm{~s} / \mathrm{step}$ and $0.125 \mathrm{eV}$ at $1.5 \mathrm{~s} / \mathrm{step}$, respectively. All of the measured binding energies were referenced to the $\mathrm{C} 1 \mathrm{~s}$ line at $285.0 \mathrm{eV}$. High-resolution spectra for the region of interest were curvefitted using XPSPEAK Ver. 4.1 (Kwok, The Chinese University of Hong Kong). Curve fitting was performed using GaussianLorentzian shapes with a Shirley-type background correction. ${ }^{29}$

\section{XANES}

X-ray absorption spectra were measured at BL6.3.1, which is an entrance-slitless bend-magnet beam line equipped with a Hettrick-Underwood type varied line space (VLS) grating monochromator at the Advanced Light Source (ALS), Lawrence Berkeley National Laboratory. ${ }^{30}$ During the measurement, the ALS operated at $1.9 \mathrm{GeV}$ between 200 and $400 \mathrm{~mA}$. Solid samples were mounted on an indium foil that was fixed on a glass plate using carbon tape. We conducted XANES measurements near the $\mathrm{N} \mathrm{K}$ absorption edge in the sample chamber under the condition of room temperature and high vacuum ( $c a$. $10^{-9}$ Torr). Data were corrected by the total electron yield with a sample current mode. The photon energy was calibrated at $400.9 \mathrm{eV}$ of $\mathrm{N} \mathrm{K}$-edge of $\mathrm{BN}$.

\section{Calculation}

A structural model of TPP was constructed from single-crystal structural data. ${ }^{31}$ The structure models of TCPP and TSPP were optimized using the Amsterdam density functional (ADF) program package. ${ }^{32-34}$ Computational details of the DV-X $\alpha$ method have been described elsewhere. ${ }^{27}$ Figure 2 shows DV-

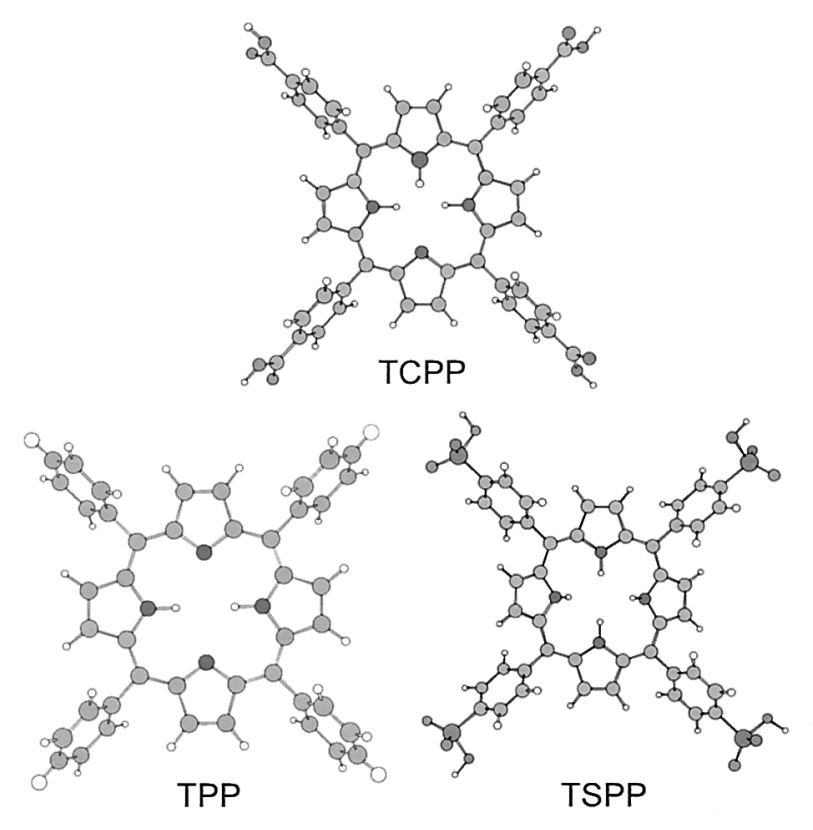

Fig. 2 Structural models of TPP, TCPP-H3, and TSPP for DV-X $\alpha$ calculations. The models of TSPP and TCPP were optimized in advance using the ADF program package.

$\mathrm{X} \alpha$ calculations that were performed using the three models (TPP, TCPP-H3 and TSPP). Numerical atomic orbitals of $1 \mathrm{~s}$, $2 \mathrm{~s}, 2 \mathrm{p}, 3 \mathrm{~s}, 3 \mathrm{p}$, and $3 \mathrm{~d}$ for $\mathrm{S}$, and of $1 \mathrm{~s}, 2 \mathrm{~s}, 2 \mathrm{p}, 3 \mathrm{~s}$, and $3 \mathrm{p}$ for $\mathrm{N}$, and of $1 \mathrm{~s}, 2 \mathrm{~s}$, and $2 \mathrm{p}$ for $\mathrm{C}$ and $\mathrm{O}$, and of $1 \mathrm{~s}$ for $\mathrm{H}$ were used as a basis set for the calculations. The DV-X $\alpha$ calculations for both models were performed using numerical integration of 1000 points per atom. The convergence of self-consistent-field iterations was set to 0.001 electrons. For simulating the XPS and XANES spectra of TPP, TCPP and TSPP, we evaluated the respective densities of states (DOSs) and the electron-transition probabilities using electron state data of MOs obtained by DV$\mathrm{X} \alpha$ calculations for corresponding models.

\section{Results and Discussion}

\section{$X P S$}

The results are shown in Fig. 3. The solid lines and dotted lines indicate fitted curves and experimental data, respectively. XPS of the TCPP showed two sharp and well-resolved peaks in the $\mathrm{N}$ 1s region, which are at $401.0 \mathrm{eV}$ and $398.9 \mathrm{eV}$ in binding energies (BE), respectively. Here, the BE values for the peak positions were determined by the fitted curves. Similarly, the XPS of TPP that was used as a reference sample also showed sharp peaks with a BE of $400.8 \mathrm{eV}$ and $398.8 \mathrm{eV}$, respectively. However, in the XPS of TSPP used as a reference sample, the only peak appeared with $\mathrm{BE}$ of $400.4 \mathrm{eV}$. Higher and lower energy peaks of TPP corresponded to protonated and nonprotonated nitrogen atoms (denoted as N1 and N2, respectively), as reported by Polzonetti et al. ${ }^{23}$ On the other hand, only the peak of TSPP corresponded to the higher peak of TPP, indicating that all of the nitrogen atoms in the porphine ring are N1. Hence, the higher and lower peaks of TCPP can be assigned to $\mathrm{N} 1$ and $\mathrm{N} 2$, respectively.

However, the ratio of the N1 to N2 peaks of TCPP in the peak area is different from that of TPP. The structure of each porphine ring for TPP and TSPP is known: the former has two $\mathrm{N} 1$ and two N2; the latter has four N1. This fact indicates that 

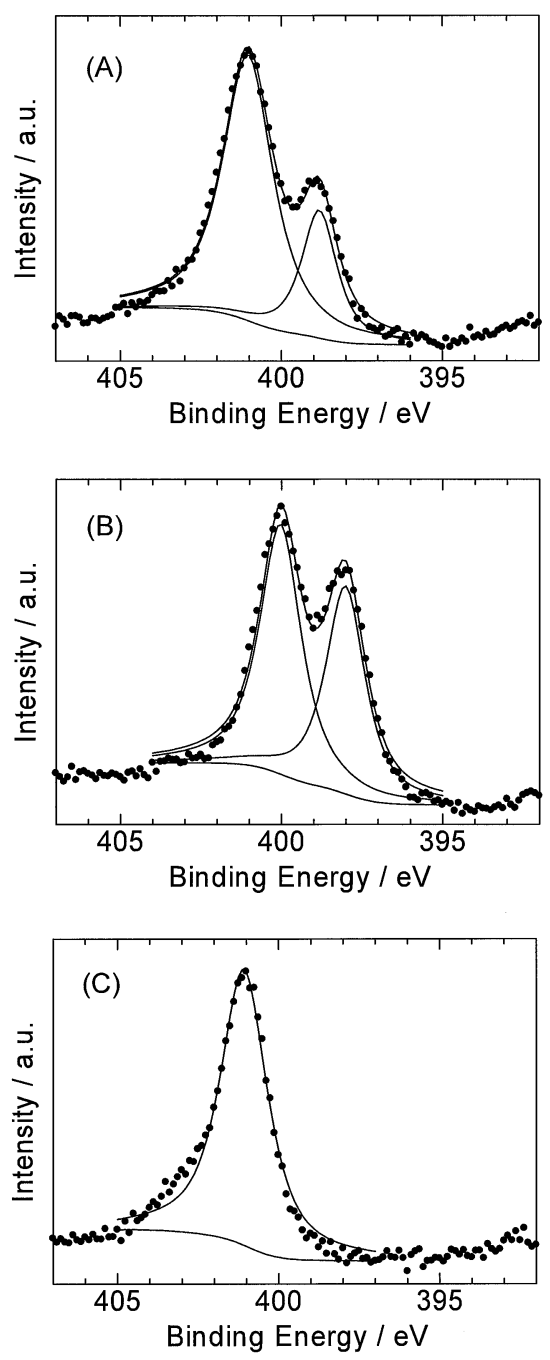

Fig. 3 Observed N 1s XPS spectra of (A) TCPP, (B) TPP, and (C) TSPP. The solid lines indicate fitted curves. The dotted lines indicate experimental data.

the ratio of the two peak areas corresponds with the ratio of the N1 species to N2 species in number: the N1 peaks of TPP and TSPP account for $56 \%$ and $100 \%$ of the total N 1s signals, respectively. Accordingly, the N1 peak of TCPP accounts for $75 \%$ of the total $\mathrm{N} 1 \mathrm{~s}$ signals; the ratio of the $\mathrm{N} 1$ species to the N2 species is estimated to be 3:1. Therefore, the four nitrogen atoms in the porphine ring in TCPP are inferred to consist of three N1 and one N2 (see in Fig. 4).

Figure 5 shows the partial density of states (PDOS) of $\mathrm{N} 1 \mathrm{~s}$ for TCPP, TPP, and TSPP, calculated using the DV-X $\alpha$ program package. The higher and lower energy peaks in each $\mathrm{N}$ 1s PDOS curve for the TPP and TCPP models are assigned to $\mathrm{N} 1$ and N2, respectively, whereas the peak in the curve for the TSPP model is assigned to N1. These results of assignments are identical with those of the corresponding experimental data. In addition, the area ratios of the N1 peak to the N2 peak for the TPP and TCPP models concur well with the corresponding experimental data, although the difference between the $\mathrm{BE}$ of the N1 and N2 signals for each of the TPP and TCPP models is larger than that of the corresponding experimental data. Therefore, the calculated TCPP model can be one structure of TCPP, which consists of three $\mathrm{N} 1$ and one $\mathrm{N} 2$ in the porphine ring (denoted TCPP-H3).

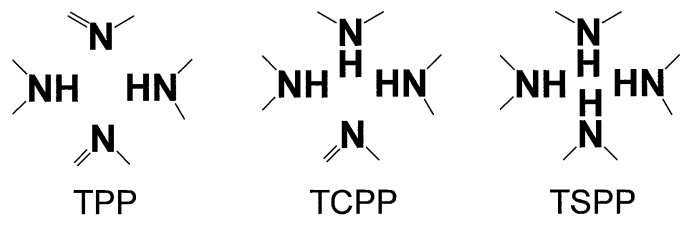

Fig. 4 Illustration of protonation for nitrogen atoms in the porphine rings of TPP, TCPP, and TSPP.
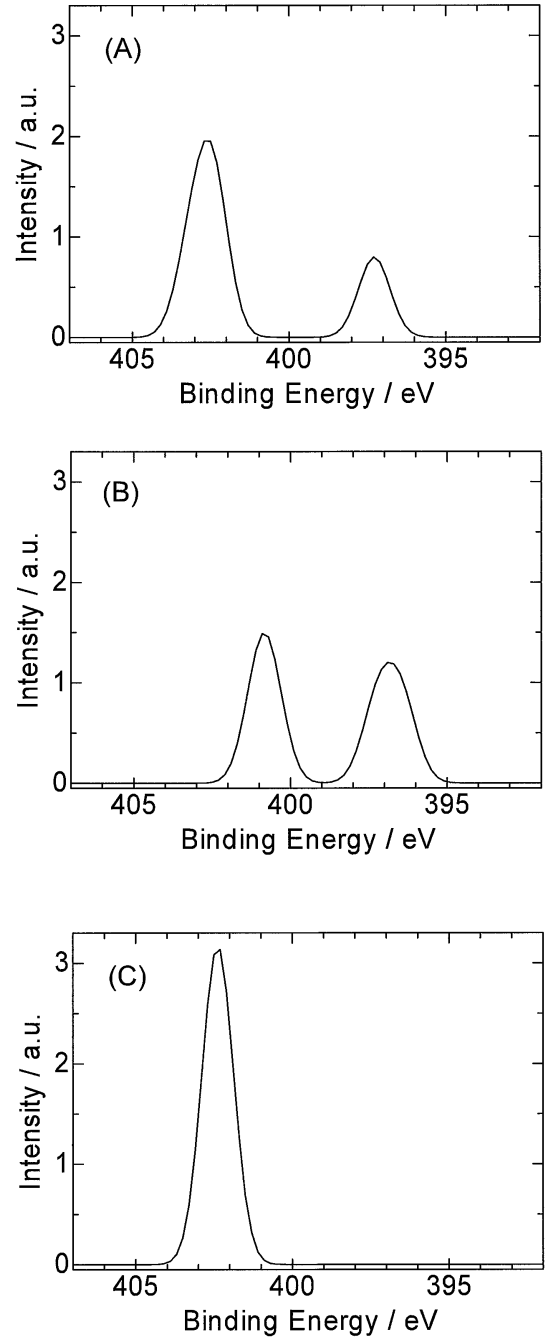

Fig. 5 Simulated N 1s XPS spectra of (A) TCPP-H3, (B) TPP, and (C) TSPP

However, the spectral shape of N 1s XPS of TCPP implies that the structure of TCPP is not a single structure, but a dual structure; the porphine ring in TCPP consists of two N1 and two $\mathrm{N} 2$ (denoted TCPP-H2), and four N1 (denoted TCPP-H4). Thus, in Fig. 3, the spectral shape of TCPP closely resembles that of TPP plus TSPP. The mixture of the calculated XPS of TCPP-H2 and TCPP-H4 is shown in Fig. 6. It appears to match the experimental spectrum of TCPP. However, using only the XPS data, it is difficult to determine whether TCPP has a single or dual structure, as explained below.

\section{XANES}

The calculated electron transition probabilities (bars) using the TCPP-H3, TPP, and TSPP models in Fig. 2 are given in Fig. 7 


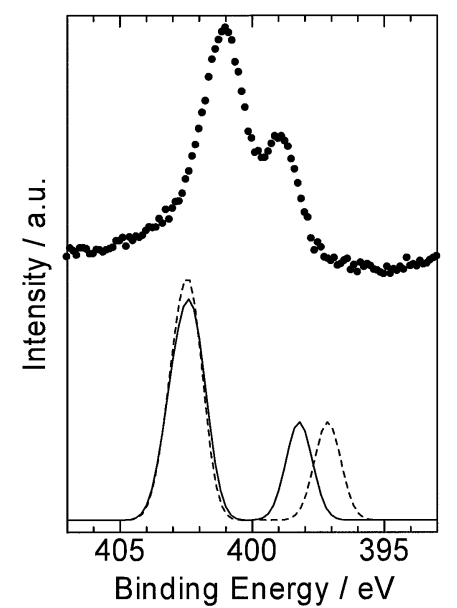

Fig. 6 Observed N 1s XPS spectrum (dotted line) of TCPP and simulated N 1s XPS spectra for the mixed spectrum (solid line) of TCPP-H2 and TCPP-H4 and the spectrum (broken line) of TCPP-H3.

along with the corresponding experimental N K XANES spectra (solid line) in the energy range, $396 \mathrm{eV}$ to $411 \mathrm{eV}$, respectively. The electron transition probabilities from the 1s orbitals of N1 and N2 to the unoccupied orbitals are divided and shown at the upper and lower sides in Figs. 7(A) - (C), respectively. The peaks that appear at approximately 398, 401, and $404 \mathrm{eV}$ on each experimental spectrum are labeled A, B, and C in order of increasing energy. The figures show that the electron transition probabilities of TPP and TSPP well express the shapes of the corresponding experimental spectra; peak A is yielded by nonprotonated nitrogen atoms (N2), whereas peaks B and C are yielded by $\mathrm{N} 2$ alone or a mixture of protonated nitrogen atoms (N1) and N2.

Both of the electron transition probabilitie patterns for N1 and $\mathrm{N} 2$ of TCPP-H3 model are more similar to those of the TPP model than to the TSPP model. Consequently, the experimental spectral feature of TCPP is presumed to be similar to that of TPP with sharp peaks at B and C. However, the experimental spectral feature of TCPP, especially that between peaks B and $\mathrm{C}$, differs from that of TPP because of the feature caused by a transition to the unoccupied level at $404 \mathrm{eV}$. This unoccupied level corresponds to peak B in the experimental spectrum of TSPP and with electron transition probabilities of N1 of TSPP model. Therefore, this result suggests that the structure of TCPP is a dual structure of TCPP-H2 and TCPP-H4, rather than a single structure of TCPP-H3.

Mixed electron transition probabilities of TCPP-H2 and TCPP-H4 are shown separately in Fig. 8 along with the experimental spectrum of TCPP to identify the dual structure of TCPP. The mixed electron transition probabilities show better agreement with the experimental spectrum than those of TCPP$\mathrm{H} 3$. Furthermore, the electron-withdrawing effect of $-\mathrm{COOH}$, which are meso-substituents in TCPP, is stronger than that of $-\mathrm{H}$ that are in TPP, but weaker than that of $-\mathrm{SO}_{3} \mathrm{H}$ that are in TSPP. For that reason, nitrogen atoms in the porphine ring of TCPP are considered to have an intermediate electron density, in contrast to TSPP, which has a higher electron density, and TPP, which has a lower electron density. Actually, the protonation constants of the free-base type of TSPP, $\log K_{\mathrm{H} 1}\left(\left[\mathrm{H}_{2}(\mathrm{P})\right]+\mathrm{H}^{+}\right.$ $\left.\leftrightarrow\left[\mathrm{H}_{3}(\mathrm{P})\right]^{+}\right)$and $\log K_{\mathrm{H} 2}\left(\left[\mathrm{H}_{3}(\mathrm{P})\right]^{+}+\mathrm{H}^{+} \leftrightarrow\left[\mathrm{H}_{4}(\mathrm{P})\right]^{2+}\right)$, are 4.60 and 4.70, respectively. ${ }^{35}$ A small difference between $\log K_{\mathrm{H} 1}$ and $\log K_{\mathrm{H} 2}$ indicates that species of $\left[\mathrm{H}_{3}(\mathrm{P})\right]^{+}$exist negligibly. Thus, it is considered that TCPP also exists predominantly as (a)

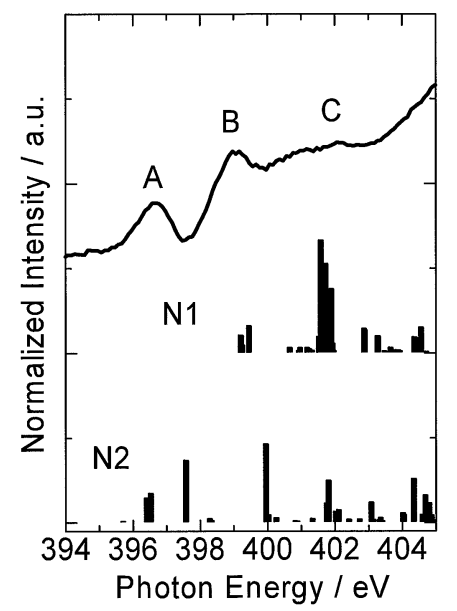

(b)

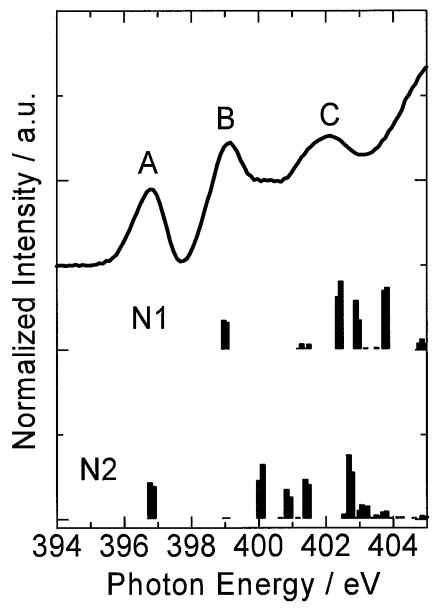

(c)

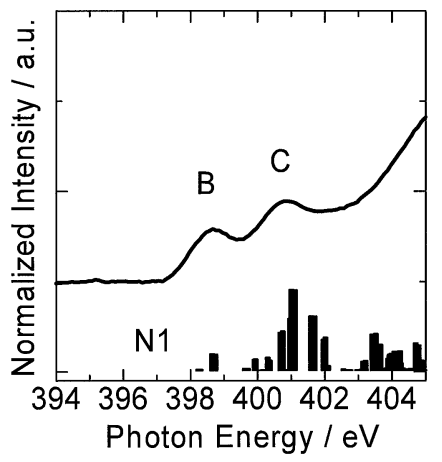

Fig. 7 Calculated electron transition probabilities (bars) and experimental N K XANES spectra (solid line) for (A) TCPP-H3, (B) TPP, and (C) TSPP. Electron transition probabilities for N1 and N2 are shown at the upper and lower sides, respectively.

two species of $\left[\mathrm{H}_{2}(\mathrm{P})\right]$ and $\left[\mathrm{H}_{4}(\mathrm{P})\right]^{2+}$. Therefore, we conclude that the structure of TCPP is a dual structure of TCPP-H2 and TCPP-H4.

\section{Conclusion}

The protonation for four nitrogen atoms in the porphine ring of porphyrin with meso-phenyl substituents was revealed for the first time using a combination of XPS and XANES. The results show that the number of protonated nitrogen atoms is related to the extent of the electron-withdrawing effect of the substituents. The number of protonated nitrogen atoms for TCPP was 


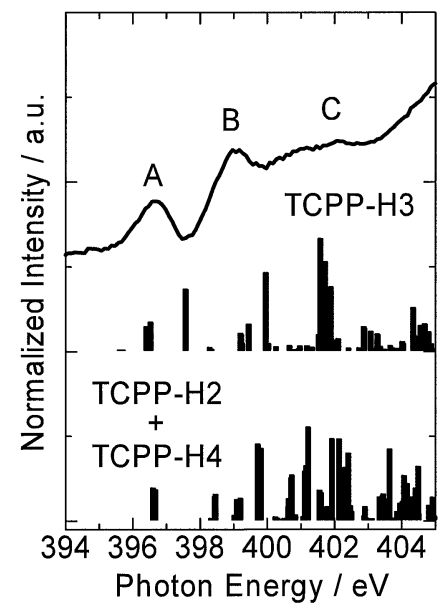

Fig. 8 Calculated electron transition probabilities (bars) and experimental N K XANES spectra (solid line) for TCPP. The electron transition probabilities for TCPP-H3 and mixed electron transition probabilities for TCPP-H2 and TCPP-H4 are shown at the upper and lower sides, respectively.

estimated as three, or a mixture of four and two from the spectrum of $\mathrm{N} 1 \mathrm{~s}$ XPS, and determined to be the latter from the spectrum of $\mathrm{N}$ K XANES. This result was also in good agreement with the protonation constants of $\mathrm{N}$ atoms in the porphyrins. Therefore, XPS data and XANES are essential probes that can be used complementarily to each other; they engender more precise results through analysis using an electron-state calculation method, such as the DV-X $\alpha$ method. For enhancing the usefulness of the combination of XPS and XAS, we are currently constructing an experimental setup of simultaneous measurements of XPS and XAS for the laboratory.

\section{Acknowledgements}

The present work was partly supported by Grants-in-Aid for Scientific Research (A) (15205012) from the Japan Society for the Promotion of Science, and by the Advanced Material Institute, Fukuoka University. The Advanced Light Source is operated by the Director, Office of Science, Office of Basic Energy Sciences, Division of Materials Science of the U.S. Department of Energy under Contract No. DE-AC0376SF00098 at LBNL.

\section{References}

1. F. L. Benedito, S. Nakagaki, A. A. Saczk, P. G. PeraltaZamora, and C. M. M. Costa, Appl. Catal. A: General, 2003, 250, 1 .

2. F. Bedioui, Coord. Chem. Rev., 1994, 144, 39.

3. F. Bedioui, J. Devynck, and C. Bied-Charreton, Acc. Chem. Res., 1995, 28, 30.

4. L. Barloy, J. P. Lallier, P. Battioni, D. Mansuy, Y. Pitfard, M. Tournoux, J. B. Valim, and W. Jones, N. J. Chem., $1992,16,71$.

5. D. Mansuy, M. A. Martinez-Lorente, and B. Battioni, $J$. Mol. Catal. A: Chem., 1996, 133, 343.
6. S. Nakagaki, C. R. Xavier, A. J. Wosniak, A. S. Mangrich, F. Wypych, M. P. Cantão, I. Denicoló, and L. T. Kubota, J. Colloid Surf., 2000, A168, 261.

7. S. Nakagaki, A. R. Ramos, F. L. Benedito, P. G. PeraltaZamora, and A. J. G. Zarbin, J. Mol. Catal. A: Chem., 2002, 185, 203

8. J. E. McMurry, Acc. Chem. Res., 1983, 16, 405.

9. B. Meunier, Bull. Soc. Chim. Fr., 1986, 4, 578.

10. I. Tabushi, Coord. Chem. Rev., 1988, 86, 1.

11. F. Montanari, S. Banfi, and S. Quici, Pure Appl. Chem., 1989, 61, 1631.

12. D. Lenoir, Synthesis, 1989, 883.

13. J. E. McMurry, Chem. Rev., 1989, 89, 1513.

14. T. C. Bruice, Acc. Chem. Res., 1991, 24, 243.

15. T. C. Bruice, Acc. Chem. Res., 1992, 25, 314

16. B. Meunier, Chem. Rev., 1992, 92, 1411.

17. R. A. Sheldon, "Metalloporphyrins in Catalytic Oxidations", 1994, M. Dekker, New York.

18. D. Mansuy, Coord. Chem. Rev., 1993, 125, 129.

19. F. Montanari and L. Caasella, "Metalloporphyrins Catalyzed Oxidations", 1994, Kluwer Academic, Dordrecht.

20. S. Y. Ma, Chem. Phys. Lett., 2000, 332, 603.

21. A. Ghosh, J. Am. Chem. Soc., 1995, 117, 4691.

22. P. G. Gassman, A. Ghosh, and J. Almof, J. Am. Chem. Soc., 1992, 114, 9990.

23. G. Polzonetti, V. Carravetta, G. Iucci, A. Ferri, G. Paolucci, A. Goldoni, P. Parent, C. Laffon, and M. V. Russo, Chem. Phys., 2004, 296, 87.

24. S. Narioka, H. Ishii, Y. Ouchi, T. Yokoyama, T. Ohta, and K. Seki, J. Phys. Chem., 1995, 99, 1332.

25. S. Mitra-Kirtley, O. C. Mullins, J. van Elp, S. J. George, J. Chen, and S. P. Cramer, J. Am. Chem. Soc., 1993, 115, 252

26. T. Okajima, Y. Yamamoto, Y. Ouchi, and K. Seki, J. Electron Spectrosc. Relat. Phenom., 2001, 114 - 116, 849.

27. H. Adachi, M. Tsukada, and C. Satoko, J. Phys. Soc. Jpn., 1978, 45,875 .

28. S. Matsuo and H. Wakita, Struct. Chem., 2003, 14, 69.

29. D. A. Shirley, Phys. Rev., 1972, B5, 4709.

30. J. H. Underwood and E. M. Gullikson, J. Electron Spectrosc. Relat. Phenom., 1998, 92, 265.

31. J. Leroy, A. Bondon, and L. Toupet, Acta Crystallogr., 1999, C55, 464.

32. G. te Velde, F. M. Bickelhaupt, S. J. A. van Gisbergen, C. Fonseca Guerra, E. J. Baerends, J. G. Snijders, and T. Ziegler, J. Comput. Chem., 2001, 22, 931.

33. C. Fonseca Guerra, J. G. Snijders, G. te Velde, and E. J. Baerends, Theor. Chem. Acc., 1998, 99, 391.

34. E. J. Baerends, J. Autschbach, A. Bérces, C. Bo, P. M. Boerrigter, L. Cavallo, D. P. Chong, L. Deng, R. M. Dickson, D. E. Ellis, L. Fan, T. H. Fischer, C. Fonseca Guerra, S. J. A. van Gisbergen, J. A. Groeneveld, O. V. Gritsenko, M. Grüning, F. E. Harris, P. van den Hoek, H. Jacobsen, G. van Kessel, F. Kootstra, E. van Lenthe, D. A. McCormack, V. P. Osinga, S. Patchkovskii, P. H. T. Philipsen, D. Post, C. C. Pye, W. Ravenek, P. Ros, P. R. T. Schipper, G. Schreckenbach, J. G. Snijders, M. Sola, M. Swart, D. Swerhone, G. te Velde, P. Vernooijs, L. Versluis, O. Visser, E. van Wezenbeek, G. Wiesenekker, S. K. Wolff, T. K. Woo, and T. Ziegler, "ADF2004.01", ed., SCM, 2004, Amsterdam.

35. K. M. Kadish, K. M. Smith, and R. Guilard, "The Porphyrin Handbook, Academic Press", 2000, San Diego. 